\title{
Implementation of the Combination of Lactation Management and Kangaroo Mother Care (KMC) Training for Village Midwives in the Sawit and Ngemplak Sub-districts, Boyolali, Indonesia
}

\section{Elia Nur A'yunin ${ }^{1}$ and Hadi Pratomo ${ }^{2}$}

${ }^{1}$ University Muhammadiyah Prof Dr HAMKA (UHAMKA), Faculty of Health Sciences, Jalan Limau II, Kebayoran Baru, Jakarta, Indonesia

${ }^{2}$ Universitas Indonesia, Faculty of Public Health, Kampus Baru UI, Depok 16424, West Java, Indonesia

\section{Abstract}

The incidence of low birth weight (LBW) in the Boyolali region of Indonesia in 2013 was 15.3 per 1000 live births, while the exclusive breastfeeding coverage was only

Corresponding Author:

Hadi Pratomo

hadi.pratomo@ui.ac.id

Received: 21 January 2018

Accepted: 8 April 2018

Published: 17 May 2018

Publishing services provided by Knowledge $\mathrm{E}$

(c) Elia Nur A'yunin and Hadi Pratomo. This article is

distributed under the terms of the Creative Commons

Attribution License, which permits unrestricted use and redistribution provided that the original author and source are credited.

Selection and Peer-review under the responsibility of the 2nd International Meeting of Public Health 2016 Conference Committee. $51.3 \%$. To resolve these problems, capacity building among midwives, through a combination of lactation management and kangaroo mother care (KMC) training, was required. The purpose of this study was to describe village midwives' experiences one month after attending the above training. This study used a quasi-experimental design with a rapid assessment procedure (RAP). Data collection was done through in-depth interviews. The informants of this study were four village midwives and pregnant and lactating women (one each). The results showed that, after one month of training, the informants had some competence in both lactation management and KMC skills. However, some informants reported some difficulties in counseling regarding breastfeeding problems. This was primarily caused by the fact that the participants did not have in-depth knowledge related to breastfeeding counseling. Breastfeeding counseling materials were not included in the above training curriculum; therefore, it is suggested that these be included in future lactation management and KMC training programs.

Keywords: Implementation of Combination of Training, Lactation Management, Kangaroo Mother Care (KMC), Village Midwive 
live births [1]. This means that about one out of every 30 children born in Indonesia died before his or her first birthday. Of these, $60 \%$ died during the first month of life, and $80 \%$ of them died between one and eleven months of age. In 2002-2012, the greatest proportion of infant deaths took place in rural areas. The predominant causes of newborns' deaths in the first week life were both low birth weight (LBW) and asphyxia [3]. UNICEF and the WHO (in DHS 2012) stated that a baby's birth weight was an important factor for determining his or her survival.

In addition, based on IDHS (2012) data of infant deaths for the previous ten years, 66 deaths of neonates and 84 deaths of infants (both per 1,000 live births), happened to babies with very LBW [1]. In the Central Java Province from 2009 to 2013, the percentage of LBW infants increased continuously, from $2.81 \%$ in 2009 , to $2.69 \%$ in 2010 and $3.73 \%$ in 2011. Furthermore, 2012 and 2013 saw a rate of $3.75 \%$, or 21,573 cases. In the district of Boyolali, there were 15.3 cases of LBW infants per 1,000 live births [2]. Both the prevention of and care for babies with LBW must be improved.

Another risk factor affecting babies' health is that of exclusive breastfeeding. The WHO recommends exclusive breastfeeding for the first six months of life. In Indonesia, based on IDHS data from 2012, only $42 \%$ of babies received exclusive breastfeeding in their first six months [1]. In the Boyolali district, the exclusive breastfeeding coverage rate in 2013 was 51.3\%, which was below the total average coverage for Central Java $67.95 \%$ [2]. Meanwhile, the achievement target of the Ministry of Health Strategic Plan (2010-2014) was for 80\% of babies 0-6 months to receive exclusive breastfeeding.

The role and contribution of health workers in promoting exclusive breastfeeding is vitally important. A study by Ranisatuhu (n.d.) indicated a positive correlation between the quality of exclusive breastfeeding communication between the health educator and mothers' knowledge of the practice. The correlation value was 0.498 ( $p=0.001<$ 0.055) (Ranistuhu n.d.). Some of the health workers who often interact with pregnant women are midwives. Another study, by Raharjo (2014), showed that midwives' roles have a significant influence $(p=0.001$ ) on the practice of early breastfeeding initiation and exclusive breastfeeding [11]. Therefore, it is indispensable to provide both information and skills to midwives regarding the management of LBW and exclusive breastfeeding.

Capacity-building among health personnel can be achieved by providing training to healthcare workers, especially, in this context, midwives, because they are the frontline for dealing with both mothers and babies. Many researchers have proven that training can increase the knowledge, attitudes, and skills of health personnel. For example, Ingram (2006) conducted a lactation management training in six health 
care centers in England involving 50 professional health workers [5]. Another study, by Law, Dunn, Wallace, and Inch (2007), compared knowledge with the skill to solve problems in breastfeeding involving 108 midwives in a "hands off" breastfeeding position training [8]. Moran, Bramwell, Dykes, and Dienwoodie (2000) also showed that midwives who completed a 20-hour lactation management training by WHO/UNICEF gained significantly more skills and support for breastfeeding than the control group $\operatorname{did}(p=<0.019)[9]$. This indicates that training is an effective way of improving health care workers' capacity.

A breakthrough in the proposed training is designed to solve the problem of babies with LBW and exclusive breastfeeding by combining two types of trainings, namely lactation management and kangaroo mother care (KMC) for LBW babies. This can become a model for a combination of lactation management and KMC training for village midwives. The training materials selected for the lactation management and KMC for the babies with LBW was based on concern for both exclusive breastfeeding and the high incidence of LBW babies. The notions of lactation management and KMC have a synergy of mutualism, because KMC is able to maximize the provision of exclusive breastfeeding for LWB babies. A study by Tunggal (2011) showed that the duration of breastfeeding for LWB babies with KMC is 2.08 times more often than without KMC [13]. This training combination of lactation management and KMC was developed as a three-day course, or 19 hours. It aims to have a positive effect and to spur a change in the behavior and skills of village midwives working to solve the problems of lactation management and caring for babies with LBW.

The objective of the present study was to describe village midwives' experiences with the implementation of knowledge and practice (behavior) one month after attending the training on lactation management and KMC. This training evaluation was based on a four-level evaluation model, namely reaction, learning, behavior, and results, developed by Kirkpatrick and Kirkpatrick (2005) [6]. The same study has been carried out by Horwood and colleagues (2009), who described the experience in terms of both the context and methodology of the training [4].

\section{METHODS}

This research was conducted in both puskesmas (public health center) in the Sawit and Ngemplak working areas, in the district of Boyolali. This study used a quasiexperimental design with a qualitative approach employing a rapid assessment procedure (RAP) method. The informants were selected purposively and consisted of 
training four village midwife participants in the above methods. In addition, three mothers, namely two pregnant mothers and the mother of a LBW baby, were recruited as key informants. Data collection took place between August 15 and September 30, 2014. Data collection was done through in-depth interviews using a semi-structured interview guideline. During the interviews, data were recorded, and the researcher took field notes. Prior to beginning each interview, an informed consent was obtained from each interviewee. Prior to the data analysis, a matrix of qualitative data was created, and the data were analyzed using content analysis. Ethical clearance was obtained from the Ethics Research Committee at the Faculty of Public Health, Universitas Indonesia.

\section{RESULTS}

The village midwife informants' ages were between 28 and 49 years, and they had an average career length of 16.71 years. All participants (100\%) stated that they had never attended either a lactation management or $\mathrm{KMC}$ training. The ages of the key informants (both pregnant women and the mother of a LBW baby) were between 22 and 50 years; they each had a high school education and were housewives.

All village midwife informants expressed the opinion that the transfer of knowledge of lactation management and KMC was based on the needs of their clients. They acknowledged that they provided information regarding breastfeeding and lactation preparation to their clients during the antenatal care visits, pregnant mothers' classes, and during delivery. Meanwhile, the transfer of knowledge of lactation management by the village midwives to the voluntary workers (cadres) and members of the Program Kesejahteraan Keluarga (PKK, Family Welfare Movement) took place in the Posyandu (Integrated Health and Family Planning Post) as a part of its breastfeeding-promotion campaign.

Each of the village midwife informants recalled her practical experience of disseminating KMC materials when she encountered mothers with premature babies, or when dealing with pregnant women who were at risk of delivering a premature or LBW baby. For example, they provided information regarding KMC for a pregnant woman at risk of preeclampsia or ones at risk of delivering a baby with intrauterine retarded growth.

"Information regarding kangaroo mother care or KMC is given when a mother [is] having a low birth weight baby or a mother who has a risk of LBW baby. It primarily depends on the case. If a mother has a complaint of breastfeeding, then the breastfeeding information is given. If a mother is complaining relevant 
to [a] low birth weight baby, then KMC information is given. But both breastfeeding and KMC information was given to both voluntary workers and to the Family Welfare Movement members].

(Village midwife informant 1)

The mother of the LBW baby confirmed the above statement. Her baby weighed 1,500 grams and used an incubator at home. She had already received a health education session and mentoring from one of the village midwives. Then, she switched in caring her baby from an incubator to natural KMC.

"I was told by the village midwife, Anik, [and] now I know about the KMC. Mrs Anik tought me about KMC in detail. I prefer to implement KMC; it is more comfortable than an incubator."

(a mother of a LBW baby, Key informant 2)

One of the supporting factors for disseminating breastfeeding information was the availability of a real example of successful breastfeeding mothers in the midwives' working environment. Two of the four village midwife informants expressed this opinion. One of the other informants mentioned that repeating breastfeeding information from the television served as another supporting factor. The fourth midwife informant mentioned that the community, in general, was aware of breastfeeding.

"This is why. Becouse the community would like to know about breastfeeding and, on TV, it was repeatedly promoted." (Village midwife informant 1)

What are the barriers to breastfeeding and lactation implementation? Three out of four village midwife informants acknowledged that they faced a problem of implementing breastfeeding among working mothers. In their area, there are a lot of manufacturing firms that employ women. They faced the problem of working mothers who stopped breastfeeding and switched to infant formula. This took place most often when the working mothers completed their maternity leave. According to the village midwife informants, the breastfeeding mothers mostly lacked intention and confidence. In addition, their working environment was not conducive to breastfeeding. The informants further noted that breastfeeding mothers mentioned the problem of expressing breast milk. Finally, two midwives indicated the unavailability of family support contributed to mothers' decisions to quit breastfeeding.

"The breastfeeding working mothers proposed a variety of reasons to quit breastfeeding. Although they are already given counseling, eventually they 
stopped breastfeeding their babies due to being too busy, [and] to not know[ing] how to continue breastfeeding. Well, they chose giving infont formula to their baby. I was also disappointed, because I did a long, detailed explanation of breastfeeding preparation. Finally, it failed in exclusive breastfeeding. When the maternal leave stops, so does the breastfeeding." (Villoge midwife informant 1)

Regarding the dissemination of KMC information, there were three midwife informants with LBW clients. None of them reported any problems with giving KMC information to these clients. Unfortunately, two of the village midwives reported a barrier in both educating and practicing KMC with their clients, because the mothers were afraid of holding a small, LBW baby. Both mothers and their families were afraid that the small neonate would become smaller, they feared the baby would fall, and they found the baby's feet disproportionate to its size. They also expressed that the KMC technique was an unusual way of carrying a small baby.

"For transferring the KMC knowledge, there was no problem. Both of the low birth weight mothers and their families feared the baby becoming smaller or tinier." (Villoge midwife informant 2)

The supporting factors when introducing KMC to clients were discussed with the midwife informants. There were several factors that eased the midwives' ability to introduce and practice KMC with the target audience. Each of the village midwife informants indicated that, apart from giving information on KMC to the mothers, the support provided by both voluntary health workers and village leaders was indispensable. The village leaders endorsed and supported the use of KMC for the LBW babies. Meanwhile, the voluntary health workers kept reminding the LBW mothers to keep practicing KMC for their LBW babies. One of the midwives pointed out that working together with voluntary health workers and village leaders was very supportive for conveying the messages to the community. The village leader had a close relationship with his community members, so if he provided new information, it was likely to be followed.

"Usually, the village midwife was not a native of the village, she came from outside of the village. The wife of the village leader usually was a local native, so the people trusted her. So this was a new method for [o] low birth weight baby. After practicing the KMC, the weight gain in a week was significant. The village leader helped to endorse the KMC as a new method. Usually in the village they were myths and taboos, in the old days, they cared for the LBW [babies] 
with a heating lamp. I explained to the people and worked together with [the] voluntary health worker who happened to be the wife of the village leader. The people were easy to accept new ideas about KMC."]

(Village midwife Informant 4)

\section{DISCUSSION}

Analysis of the results of the implementation experience of the village midwife informants showed that there were some competencies gained after attending the lactation management and KMC trainings. This can be seen from various actions taken by the village midwives in educating lactation management and $\mathrm{KMC}$ in the community. These efforts took place both in the health volunteer meeting forum and the Integrated Health and Family Planning Services "(Posyandu)". The informants gave specific information on breastfeeding was given to pregnant during antenatal examinations and classes for pregnant women.

Delivering information to the target audience was usually done during Posyandu events and the pregnant mothers' class, but the majority of the informants more often and comfortably provided information when the target audience was having an examination, or through individual counseling. This was because, with face-toface counseling methods, the informants could give more detailed information to the women. The target audience also felt that their complaints and problems received more attention, which increases their acceptance of the information. This is because empathy (feeling what others feel) is a skill that must be owned and created using interpersonal communication [10]. Personal communication makes it possible for practitioners to experience this more.

Informants' increased competency can also be seen in their KMC education efforts for society as a whole. After training, informants were more responsive to the cases of LBW babies and pregnant women with intrauterine growth-retarded infants. The practitioners helped to regularly give instructions to these mothers for evaluating their nutrition. They also became more responsive to mothers at risk of having LBW babies, such as those with preeclampsia, so they could provide information about LBW and KMC as a solution. They also practiced KMC directly when they encountered premature babies. Moreover, patients could work with practitioners to simulate KMC with a doll. These competencies are some that we expected from the training combination. The results showed that the combination training of lactation management and KMC could be applied to solve some babies' and mothers' problems. 
$\mathrm{KMC}$ information was delivered directly by practicing it with pregnant women and mothers of LBW babies. This method was an effective experience for the practitioners because of the use of direct explanation and KMC practice, so the mothers could see the real actions needed. The more senses that receive data, the more and clearer knowledge is received. Learning by doing involves more senses than by hearing alone, so this as an effective and comprehensive training approach. This is the same result as found in Kusumaningsih's 2012 research, which showed an increase in breastfeeding skills among mothers after receiving health education about KMC and the exclusive breastfeeding of LBW babies [7].

Another increased competency found among the informants was the skill to communicate KMC. This appeared when they created a cooperative plan with the voluntary health worker and village leaders to introduce KMC to society. The cooperation with the village leader came from him recommending KMC practices among the community and campaigning for it, accompanied by the health volunteer. The previous method for dealing with LBW babies was to use a heat lamp. By involving the village leaders in introducing KMC to the community, it was more accepted by the common people. This result showed a good learning process related to the KMC materials and communication during training, which helped the informants to involve and optimize the village leaders in informing others about KMC.

The village leaders serve as valuable personal references, as they that have influence and are respected by the people. Their suggestions or statements get a lot of attention, especially in regard to something relatively new to the society. This is similar to the attitude theory WHO notes, which relies on personal references. Personal references are important people who have influence on the community. In this case, the personal references are the village leaders [10].

The analysis results of the informants' experiences also revealed some obstacles. The obstacles were informants' low motivation to promote exclusive breastfeeding. They easily felt despair when facing the problems of working mothers who struggle to sustain their breastfeeding practice. In fact, this should not be an obstacle, but rather a challenge for them to succeed with this behavior. Based on the materials of the combination training on lactation management and $\mathrm{KMC}$, we must note that materials on how to counsel for exclusive breastfeeding were not given to participants. This may possible make informants less motivated and easily inhibited by the idea of promoting exclusive breastfeeding behaviors. 


\section{CONCLUSIONS}

It seems that the training participants did gain some competencies after attending the training. Participants were more responsive to lactation management services and the possibility of succeeding with exclusive breastfeeding. They were more responsive to and caring when facing LBW babies, and they were able to share information to help other mothers to implement KMC. The other competency visible in KMC communication was the involvement of social figures. Some participants felt some difficulties when providing counseling and promoting the exclusive breastfeeding campaign. This may have been because the participants did not have deep knowledge about exclusive breastfeeding, and no materials on exclusive breastfeeding were included in the above-detailed combination training curriculum.

\section{RECOMMENDATIONS}

Since the village midwives did not have deep knowledge related to exclusive breastfeeding counseling, it would be better if materials on the topic were added to the next training session.

\section{References}

[1] BKKBN, BPS, and Ministry of Health (National Family Planning Coordinating Board, Central Bureau of Statistics and the Ministry of Health). 2012. Indonesio Demographic and Health Survey 2012. Jakarta: Central Bureau of Statistics.

[2] Dinas Kesehatan Provinsi Jawa Tengah (Provincial Health Office of Central Java). 2013. Profil Kesehatan Provinsi Jawa Tengah tahun 2013 (Health Profile of Central Java Province 2013). Semarang: Dinas Kesehatan Provinsi Jawa Tengah (Provincial Health Office of Central Java).

[3] Djaja, S. 2003. Cause of Neonatal Mortality and Related Health Care Systems in Indonesia, Health Household Survey 2001," Health Researcher Bulletin 331, no. 3: 155165.

[4] Horwood, C., A. Voce, K. Vermaak, N. Rollins, and S. Qazi. 2009. Experiences of Training and Implementation of Integrated Management of Childhood Illness (IMCI) in South Africa: A Qualitative Evaluation of the IMCI Case Management Training Course, BMC Pediatrics 9 no, 62: 1-9. 
[5] Ingram, J. 2006. Multiprofessional Training for Breastfeeding Management in Primary Care in the UK. International Breastfeeding Journal 1, no. 9: 1-7.

[6] Kirkpatrick, D. L. and J. D. Kirkpatrick. 2005. Evaluating Training Programs - The Four Levels. San Francisco: Berret-Koehler.

[7] Kusumaningsih, F. S. 2012. The Influence of Health Education Kangaroo Mother Care towards Skills and Mother Confidence for Giving Exclusive Breastfeeding in Low Birth Weight Babies. Master's thesis, Indonesia University.

[8] Law, S. M., 0. M. Dunn, L. M. Wallace, and S. A. Inch. 2007. Breastfeeding Best Start Study: Training Midwives in a 'Hands Off' Positioning and Attachment Intervention, Maternal and Child Nutrition 3 no. 3: 194-205.

[9] Moran, V. H., R. Bramwell, F. Dykes, and K. Dinwoodie. 2000. An Evaluation of Skills Acquisition on the WHO/UNICEF Breastfeeding Management Course Using the Pre-Validated Breastfeeding Support Skills Tool (BeSST), Midwifery Journal 16, no. 3: 197-203.

[10] Notoatmodjo, S. 2012. Promosi Kesehatan dan Perilaku Kesehatan. Jakarta: Rineka Cipta.

[11] Raharjo, B. B. 2014. Mothers Profile and Midwives Role in Early Breastfeeding Initiation, Practical. Public Health Journal 10, no. 1: 53-63.

[12] Ranisatuhu, M. n.d. Influence of Communication Quality of Maternal Care Educator and Mother's Education Level towards Exclusive Breastfeeding Knowledge Level. Skripsi. Faculty of Communication. Semarang: Diponegoro University.

[13] Tunggal, T. H. 2011. Duration of Kangaroo Method with Successful Breastfeeding in LBW. Doctoral thesis. Jogjakarta: Gadjah Mada University. 